\title{
Analyse probabiliste de la consolidation unidimensionnelle des sols
}

\section{J.-P. MAGNAN}

Laboratoire Central des Ponts et Chaussées, 58 , boulevard Lefebvre, 75732 Paris Cedex 15

\section{A. BOUHERAOUA}

Université de Tizi-Ouzou, Institut de Génie Civil. Hasnaoua, 15000 Tizi-Ouzou (Algérie)
L'article présente une étude en éléments finis de l'influence des variations dans l'espace des propriétés du sol sur le déroulement de la consolidation unidimensionnelle. Deux paramètres ont été retenus comme variables aléatoires : le module d'élasticité isotrope $\mathrm{E}$ et le coefficient de perméabilité verticale $\mathrm{k}$. Des lois de distribution lognormales ont été utilisées. L'étude probabiliste a été effectuée par la méthode de Monte Carlo. Les résultats de cent calculs de consolidation ont été analysés en termes de variabilité des tassements et surpressions interstitielles pour montrer l'influence de la structure de la variabilité du sol (par couches horizontales ou à la fois dans le sens vertical et le sens horizontal). La réduction de la variabilité des résultats lorsque le sol varie aussi dans le sens horizontal a été montrée quantitativement. La comparaison avec des solutions déterministes classiques a aussi été effectuée. Elle indique que l'évolution moyenne de la consolidation peut être reproduite par un calcul déterministe. Le traitement probabiliste ne peut donc expliquer l'existence d'un biais par rapport aux observations de terrain.

\section{Probabilistic analysis of one-dimensional soil consolidation}

The paper presents a finite element study of the influence of the spatial variations of soil properties on the development of soll consolidation. Two parameters were chosen as stochastic variables : the modulus of isotropic elasticity $E$ and the coefficient of vertical permeability k. Lognormal probability distributions were used. The probabilistic analysis was done using the Monte Carlo method. The results of one hundred finite element consolidation calculations were analysed in terms of the variability of settiements and excess pore pressures, in order to assess the influence of the structure of the soil variability (in horizontal layers or both in the vertical and horizontal directions). The variability of the results was shown to decrease when the soil properties vary in the horizontal direction, too. The results were also compared to the classical deterministic solutions. This comparison showed that the mean consolidation settlements and excess pore pressure dissipation can be obtained from a deterministic analysis. Thus, probabilistic methods cannot be used to explain the bias that are often observed with respect to field measurements. 


\section{Introduction}

La prévision de la consolidation des sols argileux compressibles est un aspect de la mécanique des sols pour lequel beaucoup d'ingénieurs ont le sentiment de ne pas faire de calculs fiables. Ainsi, les essais de laboratoire sont souvent considérés comme pessimistes et l'on admet qu'il est possible de se tromper d'un ou plusieurs ordres de grandeur sur les coefficients de perméabilité. L'application des outils de l'analyse statistique et probabiliste aux problèmes de consolidation permet de progresser dans la compréhension des divergences possibles entre le calcul et les observations.

Les publications relatives à l'analyse probabiliste de la consolidation des sols sont restées rares après les années 1970 oủ furent publiés les premiers travaux de Freeze (1977), Asaoka (1978), Chang et Soong (1979) et Asaoka et Suzuki (1979). Les études de Magnan et Baghery (1982) au Laboratoire central des Ponts et Chaussées ont comparé dans le cas d'un remblai expérimental sur sols mous les prévisions de ces méthodes de calcul avec celles de la méthode de Monte Carlo appliquée au programme CONMULT (consolidation unidimensionnelle des sols multicouches). Les travaux de Hwang (1985) ont examiné pour leur part les correspondances entre les prévisions probabilistes et les méthodes déterministes classiques. Plus récemment, Nishimura et al. (1993, 1995) se sont penchés sur l'analyse de la consolidation unidimensionnelle par diverses méthodes.

Les travaux rapportés dans cet article analysent plus particulièrement l'effet de la variabilité spatiale des propriétés du sol sur le déroulement de la consolidation. Cette question s'est posée parce que les tentatives antérieures de modélisation probabiliste de la consolidation des sols au LCPC (Magnan et Baghery, 1982) s'étaient terminées par un échec, dû à l'existence d'un biais important des prévisions par rapport aux mesures: l'amélioration des méthodes de prévision pouvait passer soit par une révision des modèles de calcul utilisés pour l'analyse déterministe classique de la consolidation, soit par une meilleure prise en compte des propriétés réelles des sols naturels, et notamment de leurs variations dans l'espace. Ces deux démarches ont été explorées parallèlement et le présent article décrit les résultats de l'analyse probabiliste.

Après un bref rappel des causes d'imprécision dans les calculs de consolidation des massifs d'argiles naturelles, l'article présente successivement les objectifs et hypothèses de notre étude, les outils de calcul utilisés, les principaux résultats des analyses probabilistes et les conclusions qu'il est possible d'en tirer pour la poursuite des recherches et pour la pratique.

\section{2}

\section{Les sources d'erreurs des calculs de consolidation des sols}

La prévision des amplitudes et vitesses de tassements des sols fins compressibles sous les charges de surface qui leur sont appliquées reste un problème complexe où l'expérience joue un grand rôle. L'importance de l'expérience est directement liée aux nom- breuses sources d'erreurs qui subsistent dans les procédures de calcul utilisées en pratique.

Les analyses de consolidation s'effectuent généralement dans un cadre simplifié : consolidation unidimensionnelle avec la théorie de Terzaghi ou consolidation radiale avec la théorie de Barron, avec des paramètres déduits des essais œdométriques, supposés constants au cours du temps et uniformes dans toute la couche. Ces hypothèses font que le tassement est proportionnel au degré de consolidation, qu'il tend vers une valeur finie et que les surpressions interstitielles sont nulles en fin de consolidation.

\section{L'amélioration des prévisions passe par:}

- le perfectionnement de l'outil de calcul, ce qui a été fait par exemple dans le programme CONMULT du LCPC (Magnan et al., 1979; Leroueil et al., 1985). Les nouvelles hypothèses de calcul tiennent compte de la forme de la courbe de compressibilité œdométrique (domaine surconsolidé et domaine normalement consolidé, pression de préconsolidation), de l'effet du temps sur les déformations (modèle de fluage des lignes de temps de Taylor-Bjerrum), des variations du coefficient de perméabilité avec l'indice des vides, de la présence éventuelle d'un peu de gaz dans les pores du sol et de la stratification du massif de sol;

- l'amélioration des méthodes de détermination des paramètres du modèle de calcul.

L'expérience a prouvé que la forme des courbes de tassement des sols compressibles au cours du temps pouvait être reproduite de façon acceptable par la solution de la théorie de Terzaghi à condition de choisir la valeur du coefficient de consolidation de façon adéquate, mais qu'il fallait au bout d'un certain temps passer progressivement à une loi de fluage logarithmique. Les pressions interstitielles ne peuvent par contre être correctement représentées. L'utilisation d'une modélisation plus précise, telle que celle de CONMULT, permet d'effectuer des calculs plus proches de la réalité. Mais, même dans le domaine d'application de la théorie de Terzaghi, il reste un problème compliqué, qui est celui de la détermination de la valeur du coefficient de consolidation "représentatif » du comportement du massif de sol dont on veut prévoir le tassement au cours du temps.

L'estimation du coefficient de consolidation a été longtemps présentée comme une opération simple, en ce sens que l'on choisit une valeur moyenne sur les paliers de chargement de l'essai cedométrique. Comme le nombre d'essais réalisés dans les reconnaissances géotechniques reste faible en général, l'attribution d'une valeur représentative au massif de sol s'effectue souvent en découpant le sol autour des niveaux d'essais, avec des formules de calcul de moyenne comme celles de Terzaghi (1939) ou d'Absi (1965) pour homogénéiser l'ensemble des couches. Le choix de la valeur déterministe du coefficient de consolidation résulte dans ce cas d'un double calcul de moyenne, par essai puis par couche (Fig. 1). Les études théoriques et expérimentales ont montré que le choix de la valeur associée à l'essai ne devait pas provenir d'un calcul de moyenne, mais plutôt du choix raisonné de la valeur correspondant au franchissement de la pression de préconsolidation, dans le domaine normalement consolidé. Mais il reste ensuite à définir ce que peut être la valeur représentative d'une couche à partir des valeurs déterminées en quelques points. C'est pour répondre à cette question que des études probabilistes ont été entreprises. 


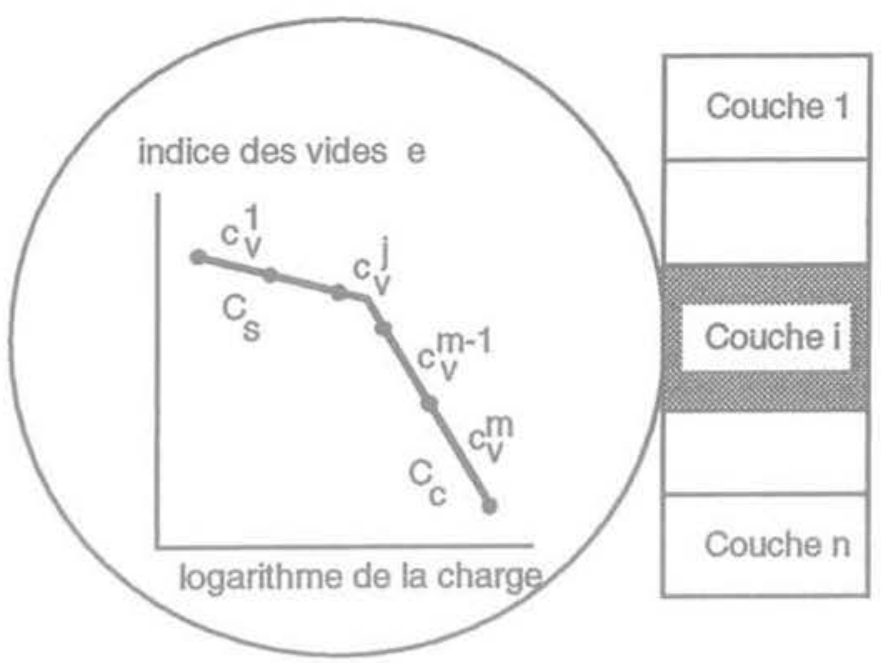

\author{
Terzaghi (1939)
}

Moyenne arithmétique des compressibilités $m_{4}$ et moyenne harmonique des coefficients de perméabilité $\mathrm{k}_{\mathrm{v} i}$ puis

$$
c_{v}=\frac{k_{v}}{m_{v} \gamma_{w}}
$$

Absi (1965)

$$
c_{v}=\left(\sum h\right)^{2} /\left(\sum \frac{h}{c_{v i}}\right)^{2}
$$

h, est l'épaisseur de la couche i,

$\gamma_{w}$ est le poids volumique de l'eau.

Fig. 1 Procédure de détermination de la valeur représentative du coefficient de consolidation dans un massif de sol pour l'application de la théorie de Terzaghi.

Procedure for estimating representative values of the coefficient of consolidation of a soil mass, for Terzaghi's onedimensional consolidation theory.

\section{3}

\section{Objectifs et hypothèses}

L'étude rapportée dans cet article avait pour objectif de quantifier l'effet de la variation spatiale potentielle des valeurs des propriétés de consolidation d'une couche d'argile sur le déroulement de la consolidation et d'estimer l'incertitude sur le résultat qu'induit l'incertitude sur les données, à modèle de calcul constant. La méthode des éléments finis a été utilisée dans cette étude, afin de pouvoir faire varier les propriétés du sol dans le sens horizontal et pas seulement dans le sens vertical, ce qui aurait pu être fait en utilisant un programme de calcul de consolidation unidimensionnelle, comme dans les travaux de Magnan et Baghery (1982).

Pour limiter le nombre de paramètres à caractériser au plan statistique, nous avons limité l'étude à un calcul de consolidation élastique isotrope, en abandonnant les possibilités offertes par la prise en compte des non-linéarités de la déformabilité du squelette dans le calcul de consolidation. Nous ne disposons pas en effet de données expérimentales sur les relations statistiques entre les différents paramètres de la courbe de compressibilité cedométrique, qui sont des variables non indépendantes. Mais surtout, comme indiqué ci-dessus, l'expérience montre que l'on peut obtenir une approximation acceptable de la courbe de tassement au cours du temps en gardant constante la valeur de $c_{v}$ dans un calcul de consolidation élastique.

Les calculs ont donc porté sur le problème de consolidation unidimensionnelle décrit sur la figure 2:

- une colonne (chomogène» de sol soumise à une charge uniforme en surface;

- des propriétés (module d'Young E, coefficient de perméabilité k) variables soit verticalement, soit verticalement et horizontalement;

- des lois de distribution statistiques de forme lognormale.

\section{Méthode d'analyse et outils de calcul}

Pour pouvoir décomposer le massif de sol soumis à une sollicitation unidimensionnelle en unités plus petites ayant des propriétés différentes dans un même plan horizontal, nous avons choisi d'effectuer des calculs en éléments finis avec les maillages représentés sur la figure 3. L'analyse probabiliste a été effectuée par la méthode de Monte Carlo, qui est la seule méthode applicable actuellement pour les calculs complexes en éléments finis.

\section{- L'outil de calcul déterministe}

L'étude a été effectuée en utilisant le sous-ensemble «Groupe 09» du système Rosalie du LCPC, destiné au calcul de la consolidation des sols par la méthode des éléments finis (Babchia et Magnan, 1986). Ce programme, remplacé actuellement par CESAR-LCPC, permettait de calculer le comportement au cours du temps (déplacements, contraintes et charges hydrauliques) d'un massif de sol saturé d'un liquide interstitiel compressible ou incompressible, dans un cadre de couplage entre l'élastoplasticité du squelette et la diffusion du liquide interstitiel, pour les problèmes de déformation plane ou à symétrie de révolution, en milieu homogène ou hétérogène, isotrope ou anisotrope. Il a été utilisê en élasticité linéaire pour simplifier les analyses probabilistes.

- La démarche probabiliste: méthode de Monte Carlo

La méthode de Monte Carlo, qui consiste à effectuer une série de simulations numériques déterministes du phénomène étudié, en utilisant des valeurs des paramètres de calcul réparties conformément aux lois de probabilité des données, a été appliquée en affectant 
pression constante

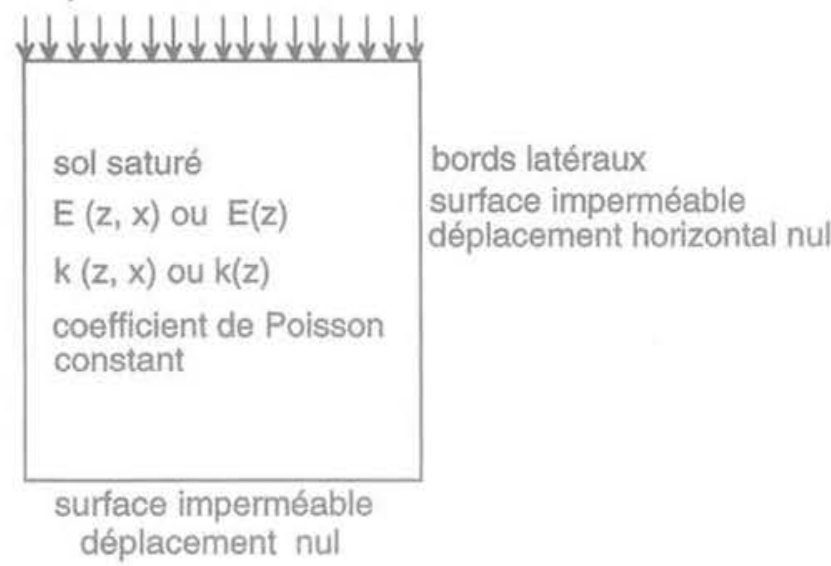

FG.2 Schéma du problème de consolidation unidimensionnelle analysé.

Main features of the analysed one-dimensional consolidation problem.

des valeurs aléatoires aux paramètres de calcul dans chaque élément du massif.

- Lois de probabilité des données du calcul

Les propriétés du sol retenues comme paramètres probabilistes du calcul de consolidation sont la déformabilité E (loi élastique isotrope) et le coefficient de perméabilité k. Pour les études présentées ici, ces deux paramètres ont été supposés indépendants. Leur choix est dicté par la variabilité importante du module d'Young et du coefficient de perméabilité $k$, et par l'influence de cette variation, soulignée par de nombreux auteurs, sur l'évolution de la consolidation.

Les données géotechniques relatives à E et à k proviennent du travail réalisé par Haghgou (1983) dans l'étude du comportement d'un remblai expérimental sur sols compressibles du site de Cubzac-les-Ponts. L'analyse statistique des valeurs de ces paramètres a donné des coefficients de variation de 0,20 et 0,70 , respectivement. Pour le module E comme pour le coefficient de perméabilité $k$, la loi de distribution lognormale semble être plus représentative que la loi normale (analyse statistique, littérature, réalité physique) et a été utilisée.

\section{- Procédure de calcul}

L'approche statistique utilisant la technique de simulation de Monte Carlo étant lente et coûteuse, nous avons développé une procédure automatique de calcul qui réduit sensiblement le temps de calcul et facilite l'analyse des résultats. Elle comporte trois parties distinctes:

1 -génération des valeurs aléatoires des paramètres $\mathrm{E}$ et $k$;

2 - réalisation séquentielle des n calculs déterministes de consolidation, avec stockage automatique des résultats en différents points du maillage:

3 -exploitation statistique des résultats.

\section{5}

\section{Données des calculs}

Nous avons calculé les tassements et les surpressions interstitielles à chaque pas de temps dans un massif homogène de sol soumis à une charge unifor-
(2)
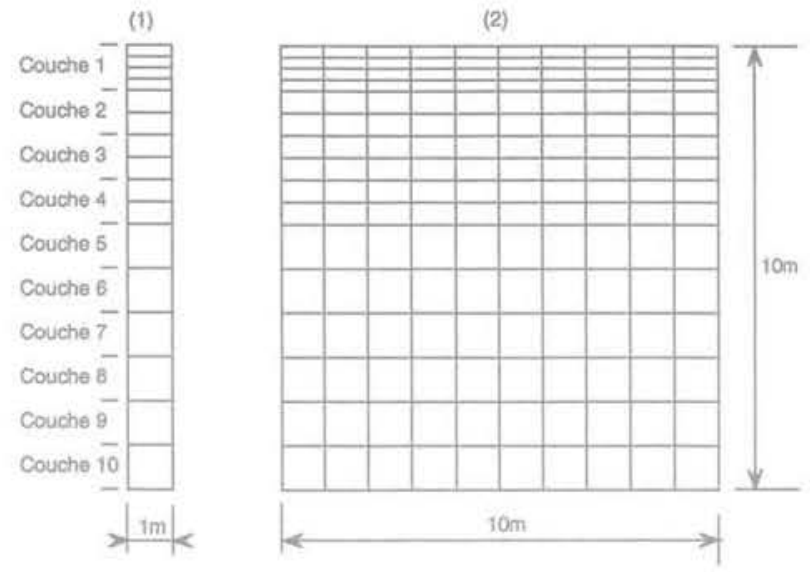

FiG:3 Maillages utilisés pour les calculs : maillage (1) pour le cas de la variabilité uniquement verticale; maillage (2) pour le cas de la variabilité verticale et horizontale. Meshes used for the finite element calculations:

mesh (1) for the case of a purely vertical variability; mesh (2) for the case of both vertical and horizontal soil variations.

mément répartie et reposant sur un substratum imperméable, dans les deux cas de:

- variabilité unidimensionnelle des paramètres E et k; - variabilité bidimensionnelle de ces paramètres.

Les conditions aux limites sont les suivantes:

- sur le bord inférieur du maillage: déplacements nuls; - sur les bords verticaux du maillage: déplacements horizontaux nuls ;

- sur le bord supérieur du maillage (surface du sol) surpressions interstitielles nulles, ce qui correspond à la notion classique de surface drainante. Aucune condition n'est imposée à la base du maillage, ce qui produit une condition d'imperméabilité à ce niveau.

La distribution initiale des pressions intertitielles est hydrostatique, avec une surface libre en haut du maillage.

Le comportement du squelette du sol est élastique et isotrope. Le sol est saturé d'un liquide interstitiel incompressible. La charge est appliquée progressivement pendant un jour et est maintenue constante jusqu'à la fin de la consolidation (20000 jours).

Cent calculs seulement ont été exécutés dans chaque cas de variabilité spatiale, ce qui ne garantit pas nécessairement la précision des conclusions de l'analyse probabiliste par la méthode de Monte Carlo. Mais la lourdeur de la démarche numérique a imposé une limitation stricte du nombre total des calculs.

Les données des calculs ont été créées au moyen d'un générateur de nombres aléatoires, sur la base d'une loi lognormale, avec des valeurs moyennes et écarts-types théoriques déduits des études d'Haghgou sur l'argile molle de Cubzac-les-Ponts:

- module d'Young:

- coefficient de perméabilité $k$ : moyenne de 0,52 MPa, coefficient de variation de $20 \%$; moyenne de $1,32.10^{-9} \mathrm{~m} / \mathrm{s}$,

coefficient de variation de $70 \%$.

Les valeurs de $\mathrm{E}$ et $\mathrm{k}$ effectivement utilisées ont les moyennes et écarts-types indiqués dans le tableau 1. Mille valeurs de chaque paramètre ont été créées pour 
TABLEAU1 Données introduites dans les calculs par éléments finis (il s'agit des distributions des valeurs fournies par le générateur de nombres aléatoires).

Data for the finite element calculations (distributions of values produced by the stochastic number generator).

\begin{tabular}{l|c|c|c|c}
\hline \multicolumn{1}{|c|}{ Calcul à variabilité unicimensionnelle } \\
\hline Paramétre & Unité & Moyenne & Ecart-type & Loi de distribution \\
Module d'Young E & $(\mathrm{MPa})$ & 0,522 & 0,105 & lognormale \\
\hline Coefficient de Poisson v & - & 0,35 & 0 & valeur fixée \\
\hline Coefficient de perméabilité k & $(\mathrm{m} / \mathrm{s})$ & $1.34 .10^{-9}$ & $0,94.10^{-9}$ & lognormale \\
\hline
\end{tabular}

\begin{tabular}{|l|c|c|c|c}
\hline \multicolumn{1}{|c|}{ Calcul à variabilitè bidimensionnelle } \\
\hline \multicolumn{1}{c|}{ Paramètre } & Unité & Movenne & Ecart-type & Loi de distribution \\
\hline Module d'Young E & (MPa) & 0,518 & 0,103 & lognormale \\
\hline Coefficient de Poisson v & - & 0,35 & 0 & valeur fixée \\
\hline Coefficient de permèabilité k & $(\mathrm{m} / \mathrm{s})$ & $1,30.10^{-0}$ & $0,94.10^{-y}$ & lognormale \\
\hline
\end{tabular}

le cas de la variabilité unidimensionnelle et dix mille dans le cas de la variabilité bidimensionnelle. Elles ont été affectées aux ( couches ») indiquées sur la fiqure 3 , dans l'ordre des couches pour le maillage (1) et des colonnes et lignes pour le maillage (2) et dans l'ordre des calculs.

Par ailleurs, nous avons effectué trois calculs déterministes, pour comparer leurs résultats à ceux obtenus dans le cas de l'analyse probabiliste: le premier utilise la moyenne arithmétique, le second la moyenne géométrique et le troisième la moyenne harmonique des coefficients de perméabilité, tandis que la moyenne arithmétique des modules est utilisée dans les trois cas. Les paramètres dont on a calculé la moyenne sont les valeurs du module d'Young et du coefficient de perméabilité créées par le générateur de nombres aléatoires en suivant les lois de probabilité de chaque paramètre.

\section{6}

\section{Résultats des calculs}

Nous allons présenter successivement les principaux résultats des calculs à variabilité unidimensionnelle et à variabilité bidimensionnelle puis les comparer.

\section{1}

\section{Variabilité unidimensionnelle}

Dans ce cas, les paramètres de calcul (module d'Young $E$ et coefficient de perméabilité k) varient en fonction de la profondeur, mais restent constants dans chaque couche au cours du temps. Ainsi toutes les hypothèses de la théorie de Terzaghi se trouvent vérifiées, sauf l'hypothèse d'homogénéité verticale.

Le massif de sol est divisé en dix couches de $1 \mathrm{~m}$ d'épaisseur, ce qui correspond au maillage de 83 nouds et 16 éléments de type Q8 (quadrilatères à 8 nœuds), représenté sur la figure 3 (1). Les points rete- nus pour l'analyse probabiliste seront désignés par la suite par leur profondeur à partir de la surface supérieure du maillage. La charge appliquée en surface est une pression uniforme de $48,3 \mathrm{kPa}$.

Une fois la série des cent calculs terminée, nous avons déterminé les moyennes du tassement et de la surpression interstitielle, pour chaque pas de temps, à différentes profondeurs, ainsi que leur écart type et leur coefficient de variation.

\section{- Résultats}

Les résultats des calculs sont résumés sur les figures 4 et 5 , consacrées respectivement aux tassements et aux surpressions interstitielles. On observe tout d'abord que la consolidation ne commence pas simultanement dans tout le massif et que les points les plus bas commencent à tasser seulement au bout de 1000 jours. Au bout de 20000 jours, le tassement est presque stabilisé et diminue linéairement avec la profondeur.

Les tassements à $0 \mathrm{~m}, 1 \mathrm{~m}, 3 \mathrm{~m}, 5 \mathrm{~m}$ et $8 \mathrm{~m}$ de profondeur ont un écart-type qui ne dépasse pas $4 \mathrm{~cm}$ au cours de la consolidation. Cet écart-type passe par un maximum à chaque profondeur. L'écart-type, initialement plus grand à proximité de la surface, évolue différemment au cours du temps en fonction de la profondeur: la position du maximum descend dans la couche.

Les coefficients de variation des tassements diminuent au cours du temps, à cause de l'augmentation du tassement moyen. Les coefficients de variation augmentent avec la profondeur dans la couche compressible, La valeur la plus faible, en surface, devient très rapidement inférieure à $20 \%$ et rapidement inférieure à $10 \%$, pour finir en dessous de $5 \%$. Ce résultat témoigne de l'effet régularisateur des variations dans l'espace des propriétés du sol, puisque les coefficients de variation des deux paramètres aléatoires (module $\mathrm{E}$ et coefficient de perméabilité k) sont égaux à $20 \%$ et $70 \%$, respectivement.

Les surpressions interstitielles moyennes à $0,5 \mathrm{~m}$, $1 \mathrm{~m}, 3 \mathrm{~m}, 5 \mathrm{~m}$ et $8 \mathrm{~m}$ de profondeur évoluent de façon 


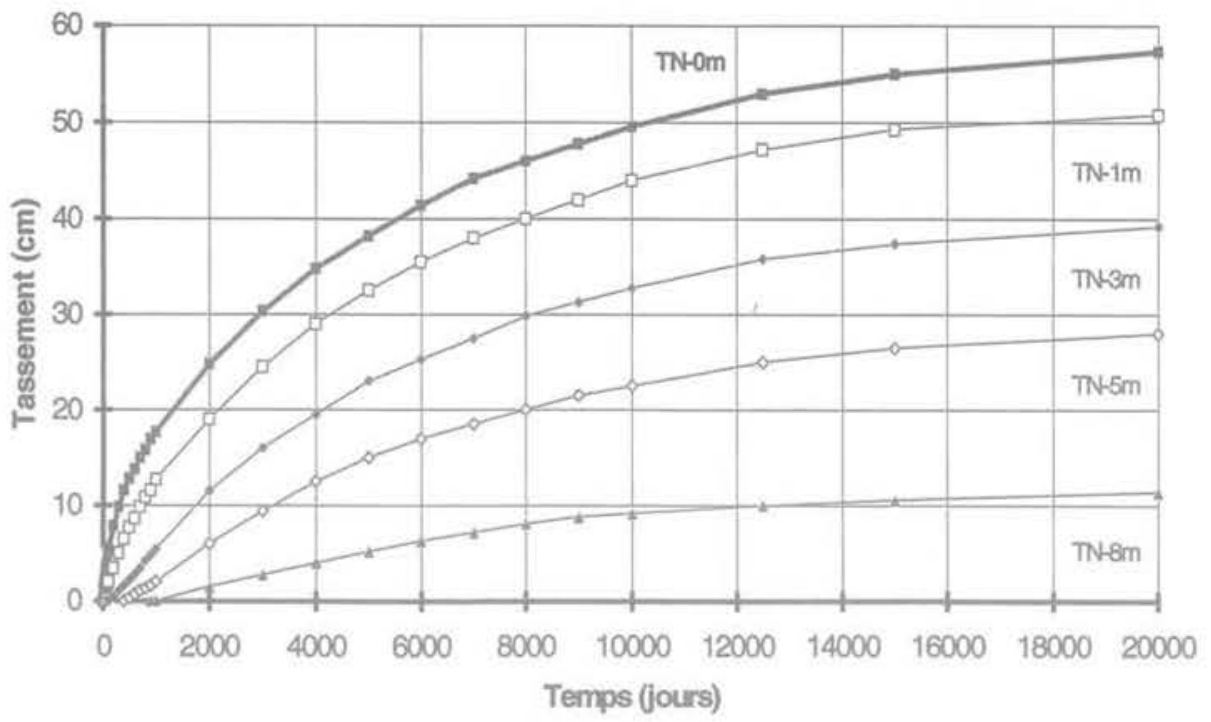

(a)

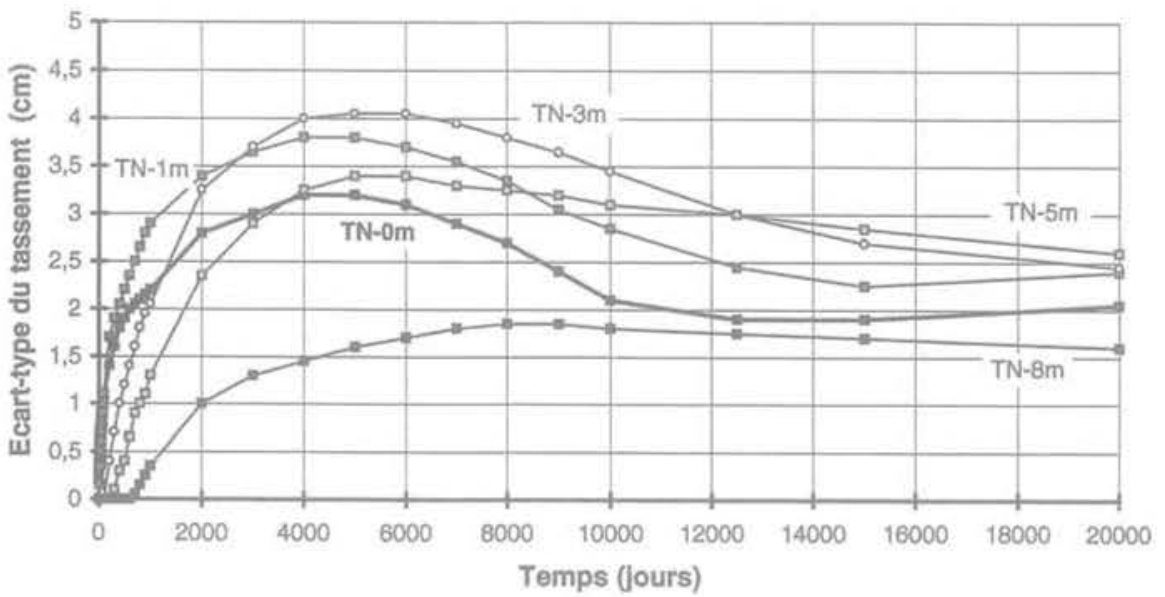

(b)

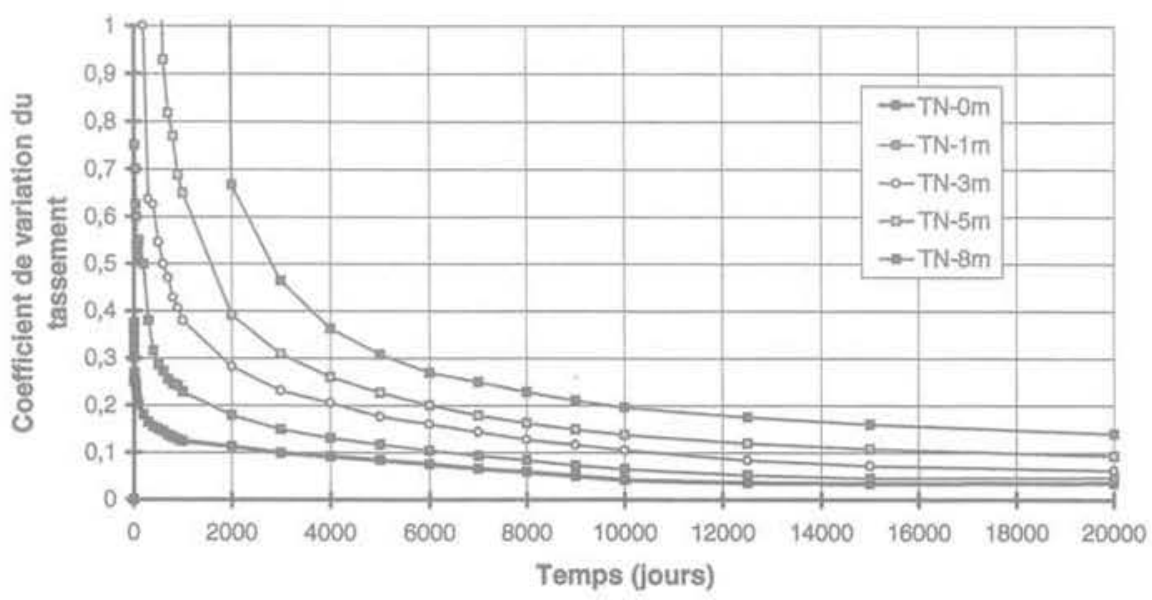

(c)

FG. 4 Évolution au cours du temps du tassement de plusieurs points du massif (a), des écarts-types (b) et des coefficients de variation correspondants (c) (variabilité unidimensionnelle).

Variations with time of the mean settlement at different depths (a), of the standard deviations (b) and of the corresponding coefficients of variation (c) (one-dimensional variability) 


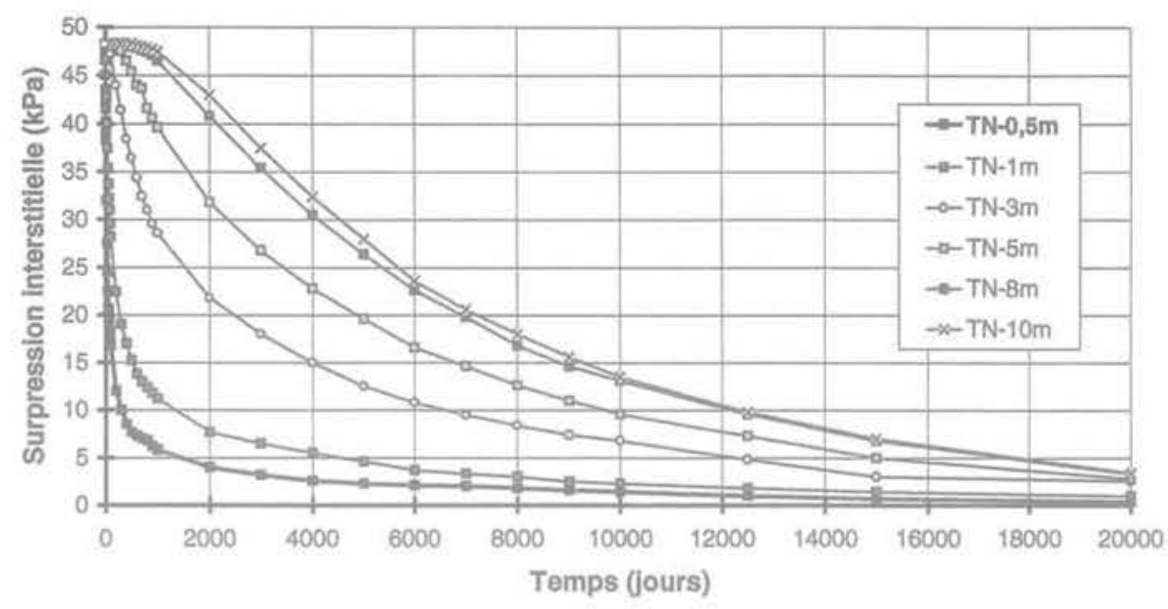

(a)

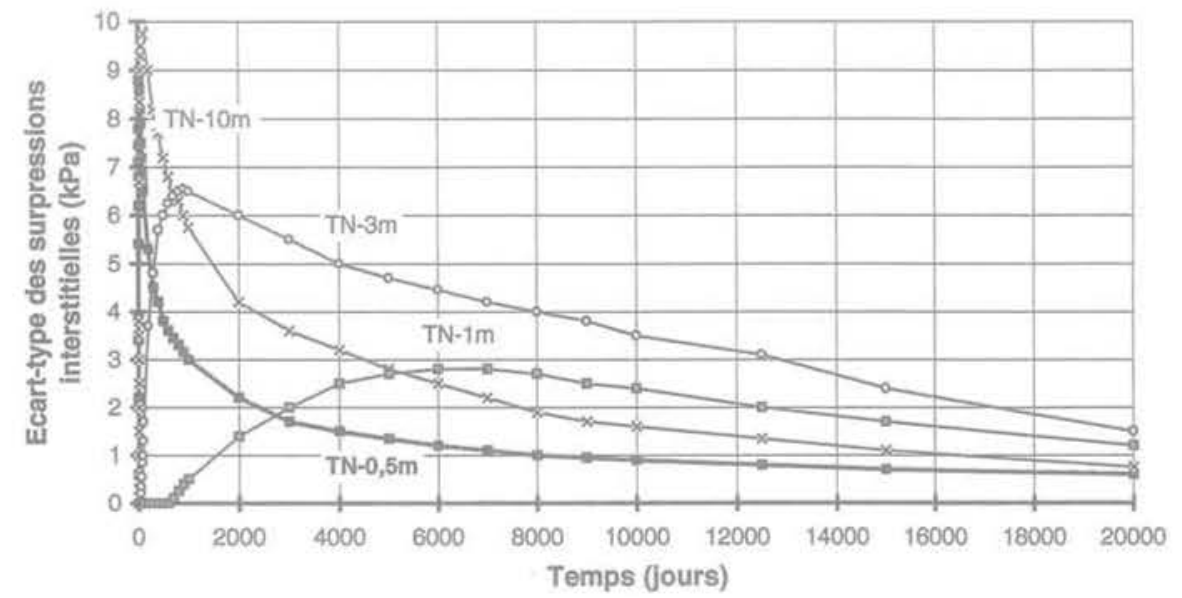

(b)

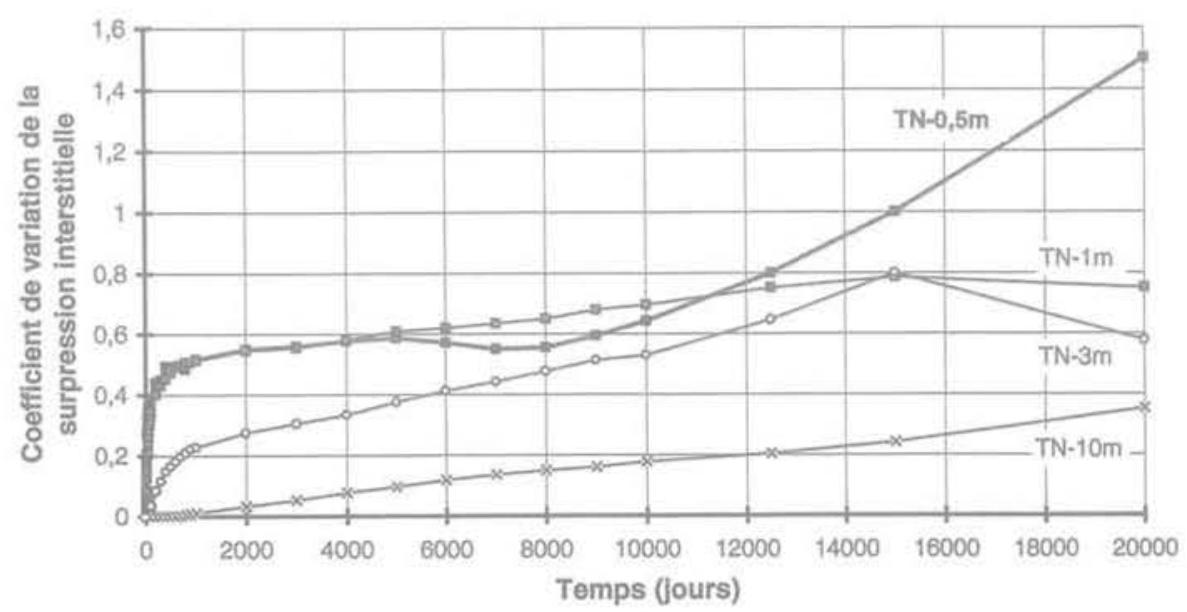

(c)

FIG. 5 Évolution au cours du temps des surpressions interstitielles moyennes en plusieurs points du massif (a), des écarts-types (b) et coefficients de variation correspondants (c)

(variabilité unidimensionnelle).

Variations with time of the mean excess pore pressures at different depths (a), of the standard deviations (b) and of the corresponding coefficients of variation (c) (one-dimensional variability). 


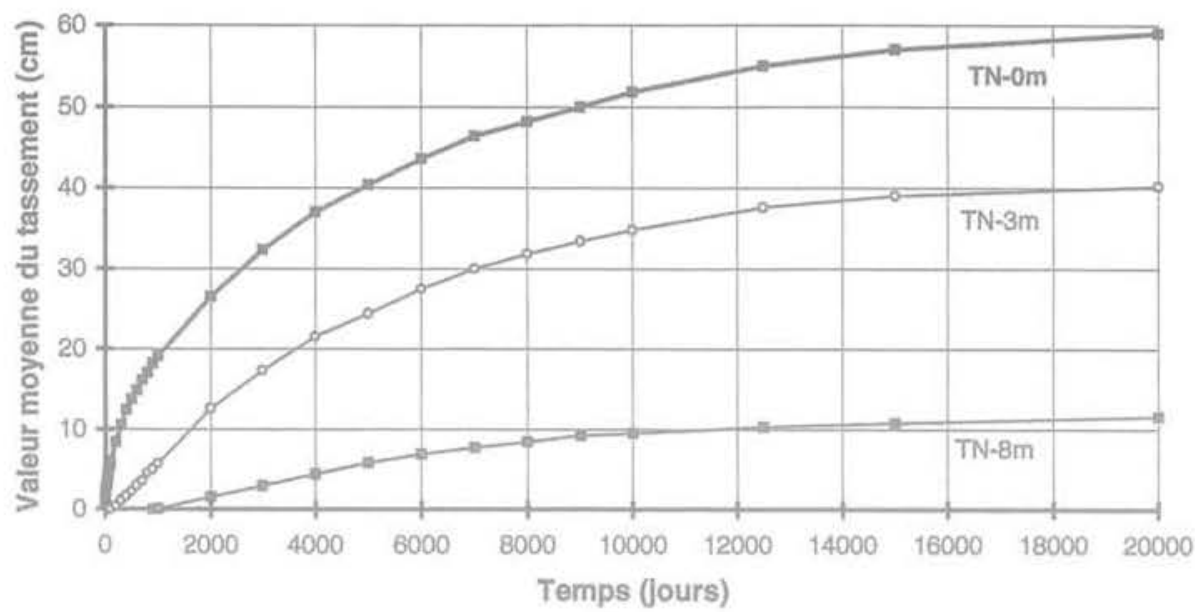

(a)

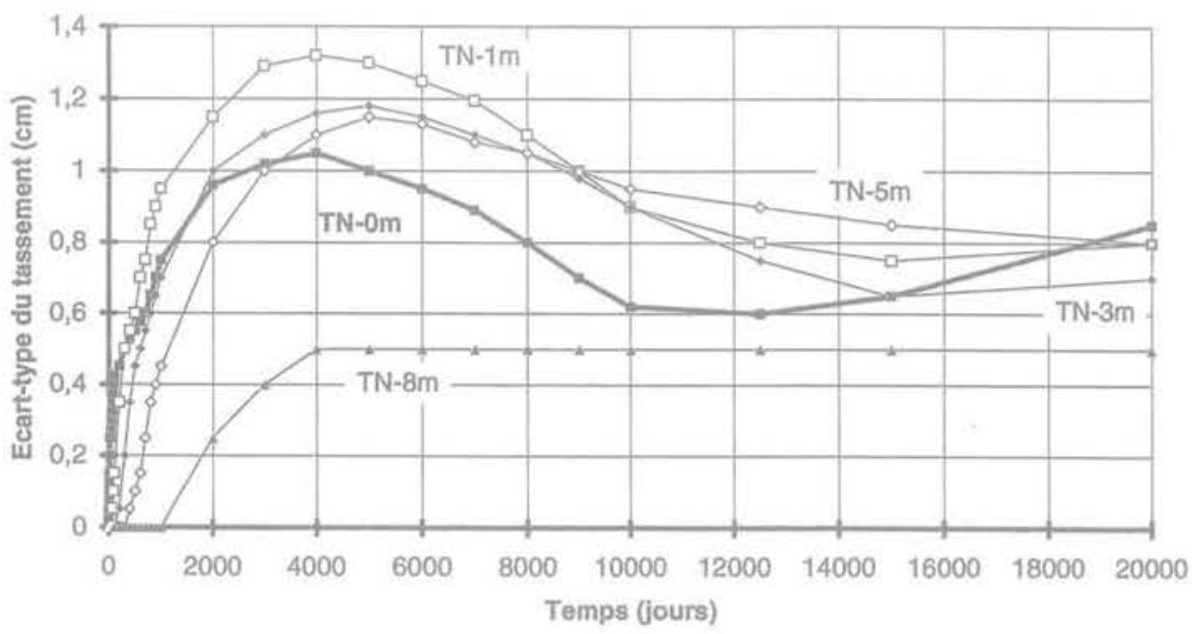

(b)

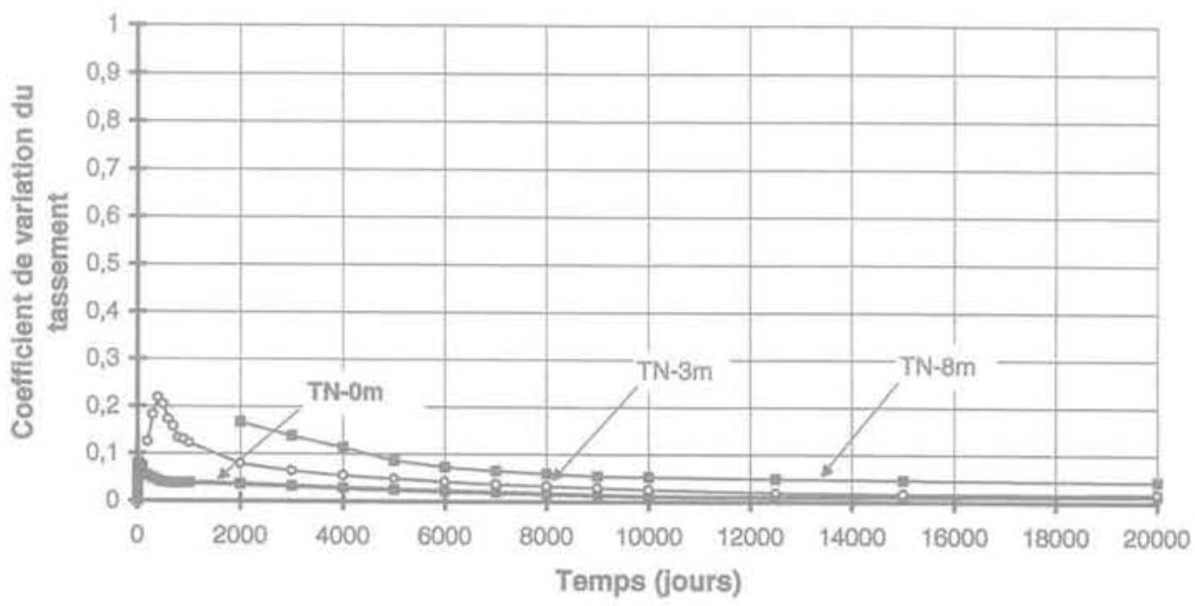

(c)

FG.6 Evolution au cours du temps du tassement de plusieurs points du massif (a), des écarts-types (b) et des coefficients de variation correspondants (c) (variabilité bidimensionnelle).

Variations with time of the mean settlement at different depths (a), of the standard deviations (b) and of the corresponding coefficients of variation (c) (two-dimensional variability). 


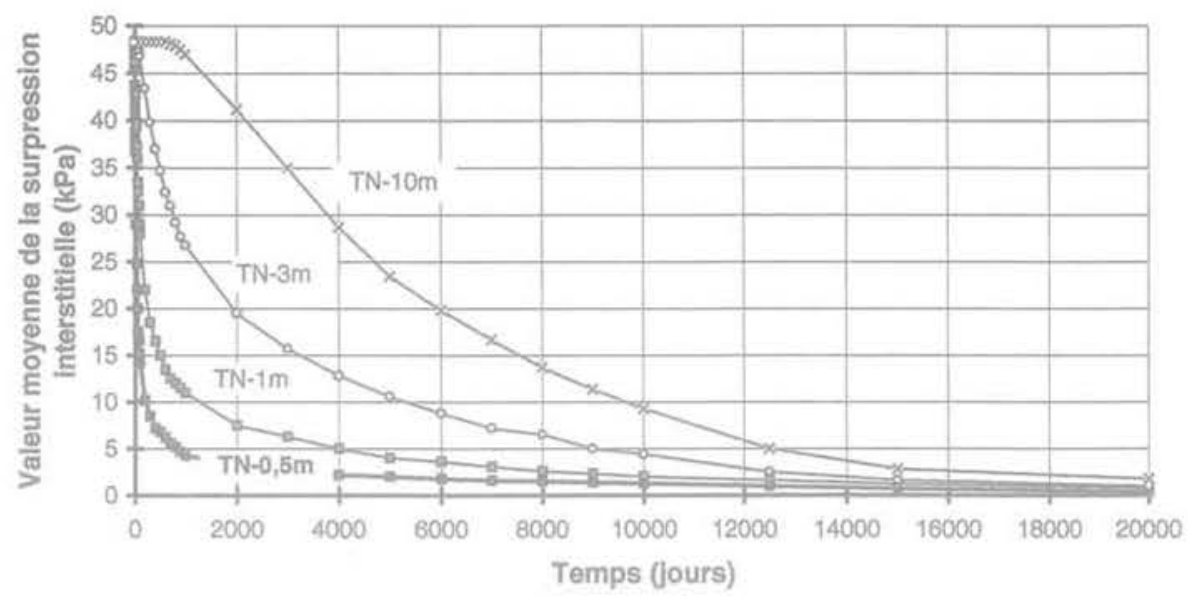

(a)

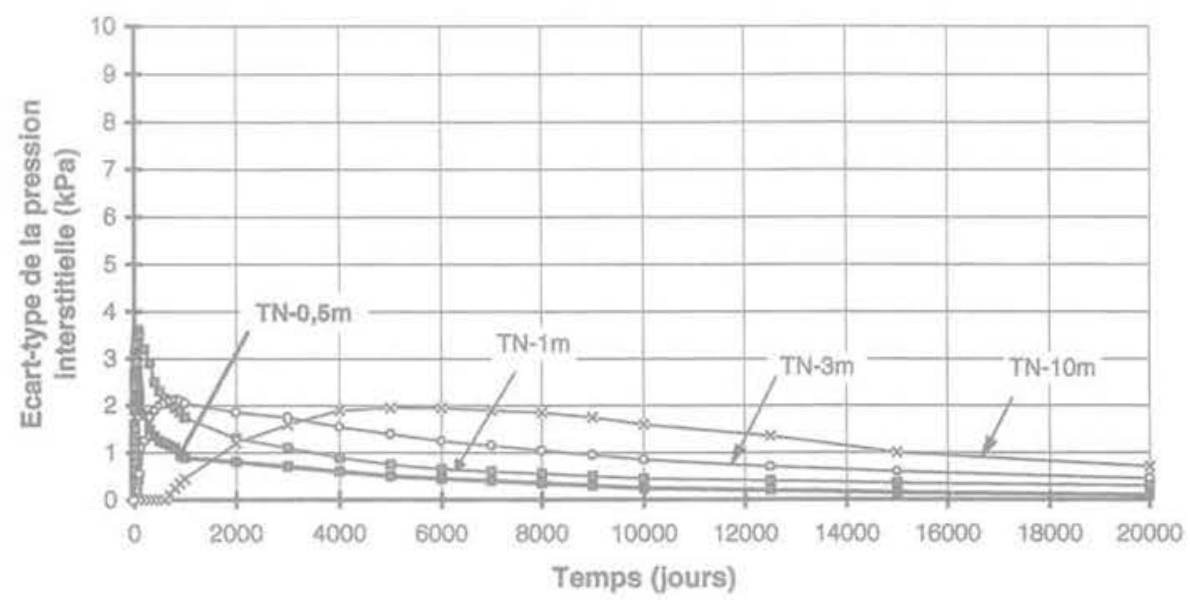

(b)

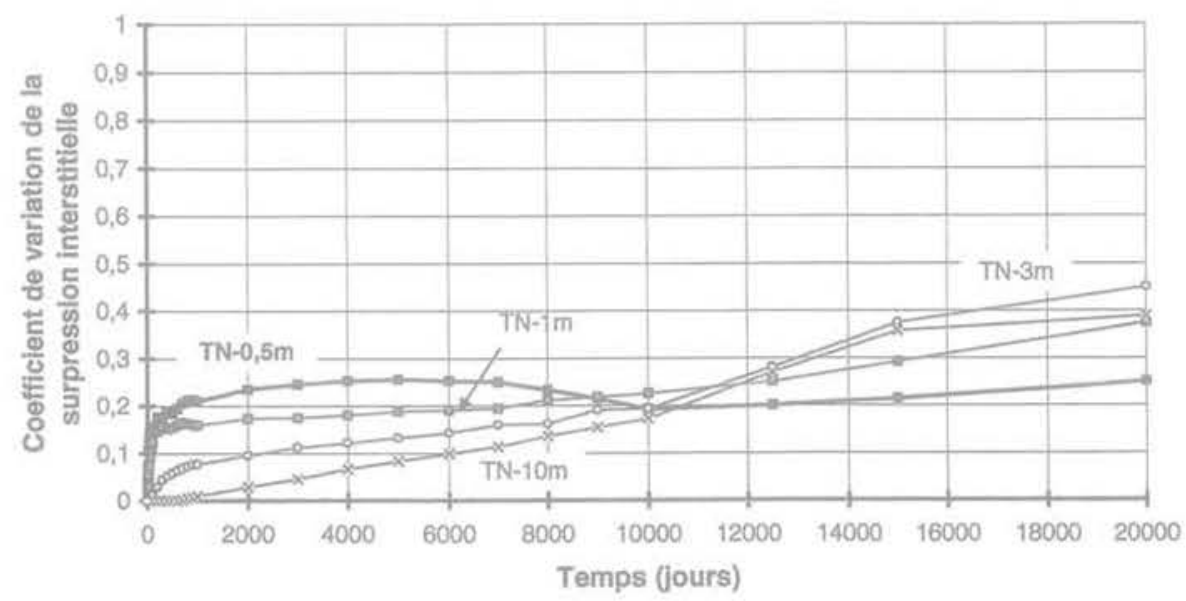

(c)

FG.7 Évolution au cours du temps de la surpression interstitielle en plusieurs points du massif (a), des écarts-types (b) et des coefficients de variation correspondants (c) (variabilité bidimensionnelle).

Variations with time of the mean excess pore pressures at different depths (a), of the standard deviations (b) and of the corresponding coefficients of variation (c) (two-dimensional variability). 


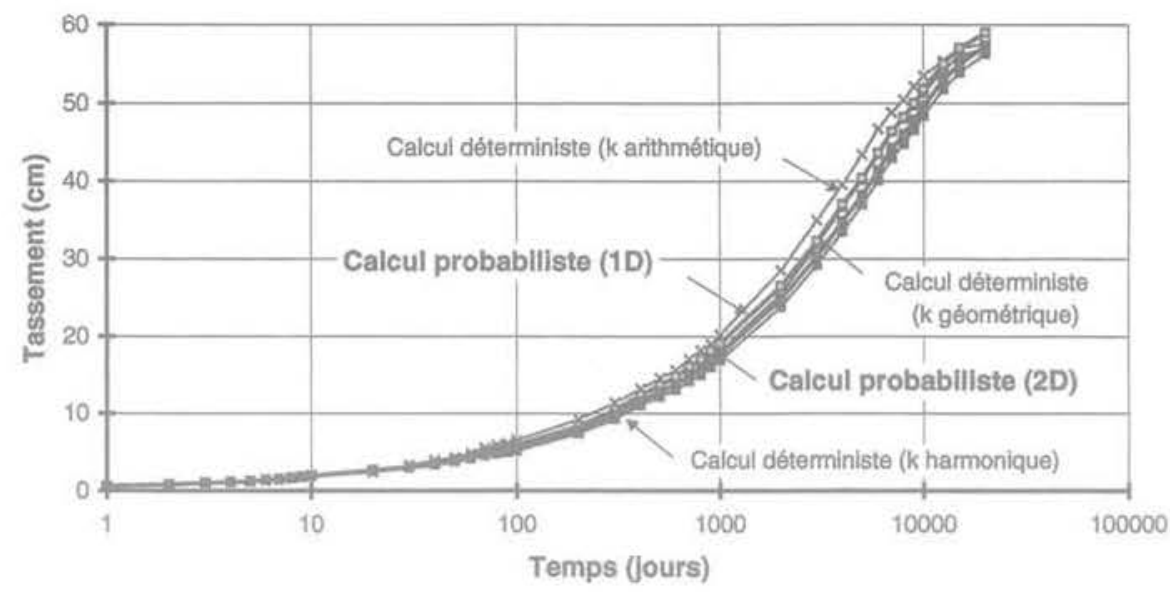

(a)

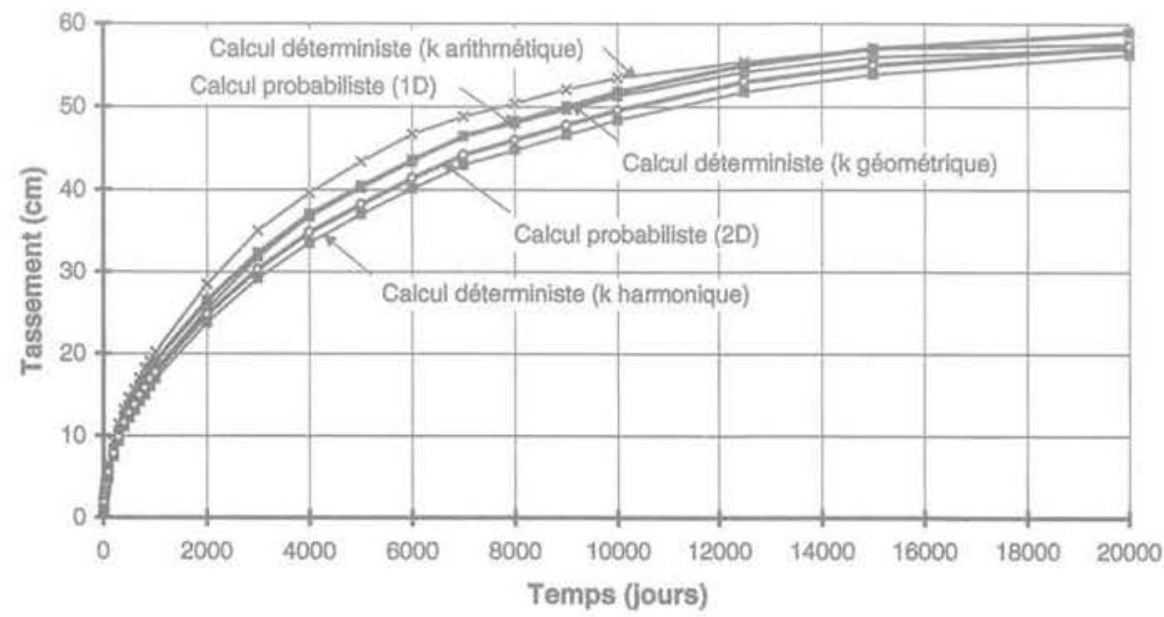

(b)

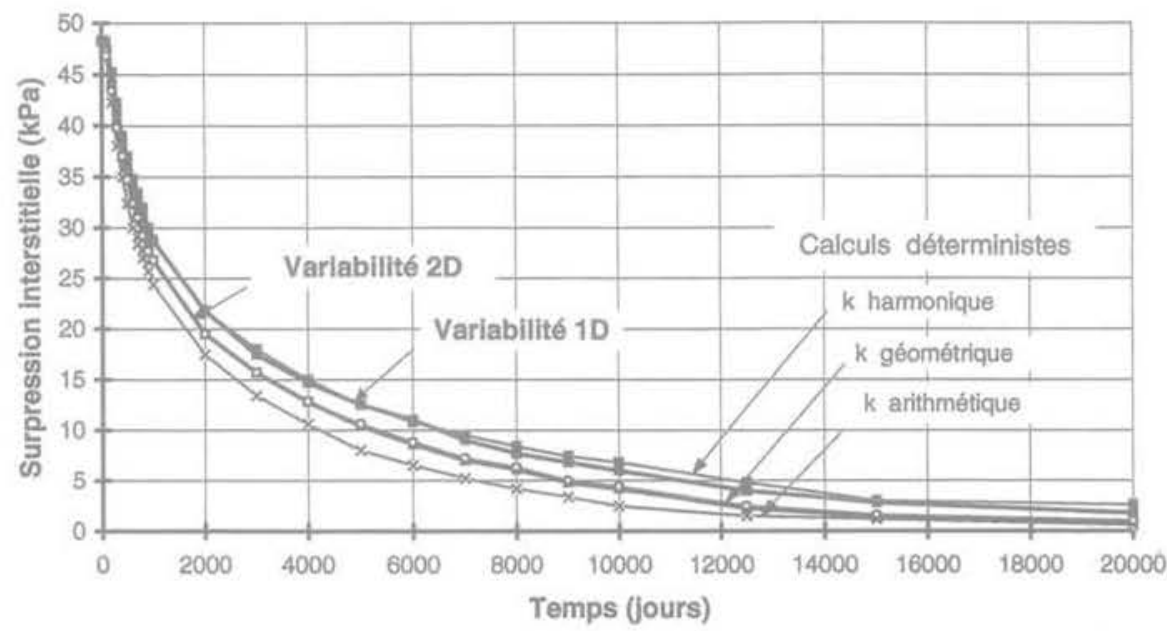

(c)

FIG. 8 Comparaison des calculs probabilistes et déterministes:

(a) Comparaison de l'évolution des tassements de la surface du sol selon les calculs (échelle du temps logarithmique); (b) Comparaison de l'évolution des tassements de la surface du sol selon les calculs (échelle linéaire du temps); (c) Comparaison de l'évolution des surpressions interstitielles selon les calculs (valeurs à $3 \mathrm{~m}$ de profondeur).

Comparison of the probabilistic and deterministic analyses:

(a) Settlement of the ground surface vs logarithm of time; (b) Settlement of the ground surface vs time (linear scale); (c) Excess pore pressures at $3 \mathrm{~m}$ depth vs time. 
régulière, comme on l'observe dans les études classiques de consolidation déterministe. Les isochrones des surpressions interstitielles présentent une forme parabolique qui tend à disparaître en fin de consolidation. On observe notamment que la dissipation des surpressions interstitielles commence au bout de 1000 jours en bas de la couche compressible et qu'il reste encore des surpressions à la fin du calcul, au bout de 20000 jours. L'écart-type de la surpression interstitielle, qui reste inférieur à $10 \mathrm{kPa}$, passe aussi par un maximum à chaque profondeur et est plus fort au milieu de la couche compressible. Le coefficient de variation reste inférieur à 70 ou $80 \%$, sauf en surface où il augmente vers la fin de la consolidation.

\section{2}

\section{Variabilité bidimensionnelle}

Dans ce cas, les paramètres du calcul E et $k$ varient en fonction de la profondeur et, dans une même couche horizontale, d'un élément (ou un groupe d'éléments) à un autre. Le massif, de $10 \mathrm{~m}$ d'épaisseur et $10 \mathrm{~m}$ de largeur, est divisé en dix couches d'épaisseur égale. Le choix de la valeur de la largeur est dicté par le besoin d'imposer un écoulement d'eau interstitielle bidimensionnel et une déformation unidimensionnelle (tassement). En effet, les déplacements horizontaux sont assez petits pour être négligés. Le maillage utilisé dans ce calcul comporte 160 éléments quadrilatères Q8 à 8 nouds et 533 points (Fig. 3 (2)).

Comme dans le cas précédent, une fois la série de cent calculs terminée, nous avons calculé les valeurs moyennes et écarts-types des tassements et surpressions interstitielles à différentes profondeurs. Les résultats de ces calculs statistiques sont rassemblés sur les figures 6 et 7 .

\section{- Résultats}

La figure 6 analyse les valeurs des tassements valeurs moyennes, écarts-types et coefficients de variation à la surface du sol, à 3 et 8 mètres de profondeur. L'allure des résultats est globalement la même que dans le calcul précédent, avec un maximum des écartstypes à chaque profondeur et une décroissance régulière des coefficients de variation après une période de croissance initiale. Les coefficients de variation sont de l'ordre de $5 \%$.

Pour les surpressions interstitielles (Fig. 7), l'allure des courbes est également classique et les écarts-types passent par un maximum à chaque profondeur, tandis que les coefficients de variation restent à peu près constants en surface (de l'ordre de $25 \%$ ) et croissent progressivement en profondeur.

\section{3}

\section{Comparaison des calculs probabilistes et déterministes}

Les deux calculs probabilistes donnent des évolutions peu différentes des valeurs moyennes des tassements et des surpressions interstitielles (Fig. 3a, 4a, 5a et 6 a). Par contre, les écarts-types et coefficients de variation sont nettement plus faibles dans le cas de la variabilité bidimensionnelle.

Les tassements moyens et surpressions interstitielles moyennes sont comparés sur la figure 8 aux courbes obtenues dans les trois calculs déterministes. Les cinq courbes de chaque figure sont assez proches, avec des écarts entre courbes inférieurs à $6 \mathrm{~cm}$ pour le tassement et à $5 \mathrm{kPa}$ pour la surpression interstitielle. Les courbes déduites des trois calculs déterministes entourent les deux courbes d'origine probabiliste, avec des tassements croissants dans l'ordre:

1) calcul déterministe avec moyenne harmonique des coefficients de perméabilité;

2) calcul probabiliste à variabilité bidimensionnelle ; 3) calcul déterministe avec moyenne géométrique des coefficients de perméabilité ;

4) calcul probabiliste avec variabilité unidimensionnelle;

5) calcul déterministe avec moyenne arithmétique des coefficients de perméabilité.

Pour sa part, la vitesse de consolidation (de diminution de la surpression interstitielle) augmente dans le même ordre, c'est-à-dire que la pression interstitielle diminue plus vite quand le tassement est plus rapide.

On observe que la moyenne harmonique des coefficients de perméabilité, qui correspond en hydraulique des sols à un écoulement perpendiculaire à la stratification du milieu, donne comme il est normal la vitesse d'écoulement et donc de consolidation la plus faible (elle privilégie les faibles perméabilités). La moyenne arithmétique, que l'on utilise pour les écoulements parallèles à la stratification du milieu, donne naturellement la valeur la plus forte des vitesses d'écoulement donc de consolidation (elle privilégie les perméabilités les plus fortes). La moyenne géométrique, qui ne correspond à aucun cas simple de stratification, donne la courbe la plus proche des calculs probabilistes à variabilité bidimensionnelle, qui nous paraissent eux-mêmes les plus proches de la réalité.

\section{7}

\section{Commentaires}

Les calculs présentés dans cet article montrent qu'il y a peu de différences entre le résultat d'un calcul déterministe réalisé avec une valeur moyenne des modules d'Young et du coefficient de perméabilité et l'évolution movenne du tassement et de la surpression interstitielle déduite des calculs probabilistes. D'autre part, les coefficients de variation autour des courbes moyennes sont faibles. Ils sont du même ordre de grandeur que ceux des modules pour les tassements et que ceux des coefficients de perméabilité pour les surpressions interstitielles dans le cas d'une variation unidimensionnelle (par couche horizontale) des propriétés du sol. Lorsque l'on suppose que le sol peut varier aussi dans la direction horizontale, ils sont encore divisés par un facteur trois ou quatre et la prise en compte d'une variabilité tridimensionnelle diminuerait encore leur valeur: Ces résultats montrent que le comportement (théorique) des massifs de sols argileux est assez proche du comportement que l'on peut calculer avec les valeurs moyennes des paramètres de compressibilité et de perméabilité.

Cette question a aussi été étudiée par Hwang (1985) et par Nishimura et al. (1995). Hwang a analysé l'influence sur le déroulement de la consolidation de cinq paramètres: indice des vides initial $e_{\alpha^{\prime}}$ indice de compression $C_{c}$, pression de préconsolidation $\sigma_{p}^{\prime}$ et indice de variation de la perméabilité $C_{k}$ [coefficient de la relation $\left.\Delta \mathrm{e}=\mathrm{C}_{\mathrm{k}} \Delta(\mathrm{lg} \mathrm{k})\right]$ qui suivent des lois normales 
et coefficient de perméabilité $\mathrm{k}$ à distribution lognormale. Cet auteur a aussi étudié l'influence de la dimension de l'analyse (calculs uni-, bi- et tridimensionnels) et des effets de réduction de variance sur les paramètres. Hwang conclut que :

- le passage de l'étude unidimensionnelle à une étude bidimensionnelle puis tridimensionnelle augmente la vitesse de tassement et diminue la variabilité des résultats. Les valeurs moyennes sont voisines des solutions déterministes et l'effet de réduction de variance lié aux dimensions du volume de sol concerné par la consolidâtion est très net;

- la perméabilité est la cause de la plus grande partie de la variabilité des résultats:

- globalement, l'incertitude sur la solution des équations de consolidation des sols due à la variation aléatoire des propriétés des sols n'est pas significative. La solution déterministe, basée sur la moyenne géométrique pour la perméabilité et la moyenne arithmétique pour les autres propriétés, est pour la plupart des problemes pratiques en bon accord avec les valeurs moyennes des solutions probabilistes.

Nishimura et al. (1995) se sont intéressés pour leur part à la consolidation unidimensionnelle d'un sol normalement consolidé dont l'indice des vides initial $e_{0}$ l'indice de compression $C_{c}$ le coefficient de perméabilité initial $k_{0}$ et l'indice de variation de la perméabilité $\mathrm{C}_{\mathrm{k}}$ sont des variables aléatoires normales corrélées deux à deux et autocorrélées en fonction de la profondeur. Leur calcul en éléments finis est réalisé sur une colonne d'éléments à variabilité unidimensionnelle au sens du présent article (variations par couches horizontales homogènes). Nishimura et al. concluent que : - les écarts-types du tassement augmentent avec la charge appliquée mais restent à peu près constants avec la profondeur et au cours du temps à partir de $50 \%$ de consolidation;

- les écarts-types de la surpression intertitielle et du degré de consolidation passent par un maximum au cours du temps. Ceux de la surpression interstitielle sont constants sur l'épaisseur de la couche;

- la variabilité du coefficient de consolidation exerce l'effet le plus important sur la variabilité des résultats. L'effet de $C_{\text {c }}$ et de $C_{k}$ augmente avec l'amplitude de la charge appliquée, $C_{c}$ ayant un effet dominant sur le tassement final.

Les résultats détaillés de nos calculs (Bouheraoua, 1989) ne permettent pas de comparaisons complètes avec ceux de Nishimura et al. (1995), mais ils montrent que les écarts-types du tassement et de la surpression interstitielle peuvent varier du simple au double en fonction de la profondeur (Fig. 4b et 5b),

\section{8}

\section{Conclusion}

Les analyses probabilistes de consolidation unidimensionnelle dans un massif de sol statistiquement homogène mais à variabilité uni- ou bidimensionnelle montrent que la variabilité du résultat du calcul est nettement plus faible que celle des données et que ce résultat est accentué par l'aspect multi-dimensionnel de la variabilité des propriétés du sol.

D'un point de vue pratique, une estimation satisfaisante de l'évolution moyenne des tassements et des surpressions interstitielles semble être obtenue par un calcul déterministe unique où la déformabilité est caractérisée par une moyenne arithmétique des données et la perméabilité par une moyenne géométrique des données correspondantes. Cette constatation implique que les écarts entre les prévisions et les mesures de consolidation des sols ne doivent pas être recherchés en priorité dans l'influence de la variabilité statistique des sols mais dans le biais (la non-représentativité) des valeurs des paramètres estimés dans le cadre des reconnaissances géotechniques.

\section{$\overline{\text { Bibliographie }}$}

Absi E. - Générallsation de la théorie de la consolidation de Terzaghi au cas d'un multicouche. Annales de ITTBTP, Paris, $n^{\circ} 211-212,1965$.

Asaoka A. - Observational procedure of settlement prediction. Solls and Founda. tions, vol. 18, n4. p. 87-101, 1978.

Asaoka A., Suzuki M. - Settlement prediction of extensive reclaimed land. Proc 3rd ICASP, Sydney, p. 477-486, 1979

Babchia M.Z. Magnan J.-P. - Analyse numérique du comportement des massifs de sols argileux. Laboratoire central des Ponts et Chaussées, Paris, Rapport de recherche LPC $n^{\circ} 140,126$ p., 1986.

Bouheraoua A - Influence de la variabilité des propriétés des sols sur la consolidation des massif́s de sols argileux. Thèse de doctorat, université Pierre-et-MarieCurie (Paris VI), Paris, 212 p., 1989.

Chang C.S., Soong T.T. - A probabilistic approach to consolidation analysis. Proc. 3rd ICASP, Sydney, p. 487-496, 1979.
Freeze R.A. - Probabilistic one-dimensional consolidation. ASCE. Journal of the Geotechnical Engineering Division, vol. 103, n GT7, p. 725-742, 1977

Haghgou M. - Etude du comportement d'un remblal expérimental sur sols compressibles. Thèse de docteur-ingénieur: université Pierre-et-Marje-Curie (Paris VI), Paris, 137 p., 1983.

Hwang D. - A probabilistic consolidation analysis for embankment foundations $\mathrm{PhD}$ Thesis, University of Maryland, $355 \mathrm{p}, 1985$.

Leroueil S., Magnan J.-P., Tavenas F. Remblais sur argiles molles. Technique et Documentation. Lavoisier, Paris. 342 p., 1985.

Magnan J.-P., Baghery S., Brucy M., Tavenas F. - Etude numérique de la consolidation unidimensionnelle en tenant compte des variations de la perméabilité et de la compressibilité du sol, du fluage et de la non-saturation. Bulletin de liai- son des Laboratoires des Ponts et Chaussées, Paris, n 103; p. 83-94, 1979.

Magnan J.-P., Baghery S. - Etude probabiliste du comportement d'un remblai sur sols mous. Revue Francaise de Géotechnique, Paris, n²0, p. 5-11, 1982.

Nishimura S. Fuiii H. Shimada K - Settlement prediction using one-dimensional consolidation analysis with special reference to variability of soil parameters and observational procedure. Proceedings, ICOSSAR'93, Innsbrück, Structural safety and reliability, vol. 3 , p. 2051-2054, 1993.

Nishimura S., Fujii H., Shimada K. - FEM consolidation analysis considering the variability of soil parameters. Proceedings, ICASP7, Paris, vol. 1, p. 85-92. 1995.

Terzaghi K., Fröhlich O.K. - Théorie de la consolidation des couches argileuses. Dunod, Paris, 1939. 